\title{
Sistemas agroflorestais e seus efeitos sobre os atributos químicos em Argissolo Vermelho-Amarelo do Cerrado piauiense
}

\author{
Bruna de F. Iwata ${ }^{1}$, Luiz F. C. Leite $^{2}$, Ademir S. F. Araújo ${ }^{3}$, \\ Luis A. P. L. Nunes ${ }^{3}$, Christoph Gehring ${ }^{4} \&$ Liliane P. Campos ${ }^{5}$
}

\begin{abstract}
RESUMO
Objetivou-se, com este trabalho, quantificar os efeitos dos Sistemas Agroflorestais com diferentes tempos de adoção e a agricultura de corte e queima sobre os atributos químicos de um Argissolo VermelhoAmarelo em áreas de cerrado do estado do Piauí. Em duas épocas climáticas foram estudados quatro sistemas: sistemas agroflorestais com seis e treze anos de adoção, agricultura de corte e queima e uma floresta nativa do cerrado. As amostras de solo foram coletadas em quatro profundidades para determinação dos atributos químicos. No solo sob os Sistemas Agroflorestais foram observados, em

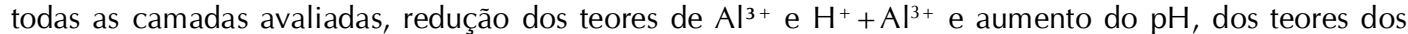
nutrientes e do carbono orgânico total em relação aos demais sistemas. No solo sob agricultura de corte e queima, verificou-se que apenas nas camadas superficiais a cinza depositada sobre o solo promoveu aumento dos nutrientes, exceto para o $\mathrm{P}$, que foi maior em todas as camadas. Nos demais sistemas houve diminuição dos teores dos nutrientes no período chuvoso. Os sistemas agroflorestais promoveram aumento dos teores de nutrientes do solo e garantiram melhoria da qualidade química do solo em áreas de cerrado no estado do Piauí.
\end{abstract}

Palavras-chave: conservação do solo, qualidade do solo, manejo sustentável

\section{Agroforestry systems and its effects on chemical attributes of an Ultisol in the 'Cerrado' of Piaui State, Brazil}

\begin{abstract}
This study aimed to quantify the effects of agroforestry systems (AFS), with different periods of adoption, and slash and burn agriculture (SB) on the chemical attributes of an Ultisol in the 'Cerrado' of Piaui State, Brazil. In two distinct climatic seasons (dry and rainy) four systems were studied: AFS with six (AFS6) and thirteen years (AFS13) of adoption, an area under SB and a native forest (NF), as a reference. In the AFS, in all depths, higher reduction in the $\mathrm{Al}^{3+}$ and $\mathrm{H}^{+}+\mathrm{Al}^{3+}$ contents were observed as well as an increase of $\mathrm{pH}$ value and contents of nutrients $(\mathrm{N}, \mathrm{P}, \mathrm{Ca}, \mathrm{Mg}$ and $\mathrm{K})$ and organic carbon in soil. In the soil under $\mathrm{SB}$, only in superficial layers, the ash deposited on the soil caused an increase in contents of nutrients, except for $\mathrm{P}$, which was higher in all the depths. There was significant seasonal effect in the contents of nutrients, reducing in the rainy season. Agroforestry systems promoted increase in the nutrient contents of an Ultisol and can be considered useful to improve soil chemical quality in areas of 'Cerrado' of Piauí State, Brazil.
\end{abstract}

Key words: soil conservation, soil quality, sustainable management

\footnotetext{
DCS/UFC, Avenida Mister Hull, Campus do Pici, B. Pici, CEP 60455-760, Fortaleza, CE. E-mail: brunaiwata@gmail.com 2 Embrapa Meio-Norte, Av. Duque de Caxias, 5650, B. Buenos Aires, CEP 64006-220, Teresina, PI. E-mail: luizf@cpamn.embrapa.br PPGA/UFPI, Campus Universitário Ministro Petrônio Portella, B. Ininga, CEP 64049-550, Teresina, PI. E-mail: asfaruaj@yahoo.com.br; lalnunes@yahoo.com.br

${ }^{4}$ CCA/UEMA, Cidade Universitária Paulo VI, B. Tirirical, CEP: 65054-970, São Luís, MA. E-mail: christophgehring@yahoo.com.br ${ }^{5}$ UNESP, Campus Prof. Júlio de Mesquita Filho, Via de Acesso Prof. Paulo Donato Castellane s/n, CEP 14884-900, Jaboticabal, SP. E-mail: licalivre@hotmail.com
} 


\section{INTRODUÇÃO}

O solo é um recurso essencial responsável pelas boas produtividades da agropecuária, pela manutenção da qualidade do meio ambiente e, consequentemente, pela sanidade de plantas, animais e seres humanos (Sharma et al., 2005). No entanto, sua utilização inadequada, sobretudo por meio da adoção de sistemas convencionais, tem ocasionado a degradação de suas propriedades físicas, químicas e biológicas (Sá et al., 2009) como, por exemplo, a desestruturação e compactação, a redução da fertilidade, a oxidação acelerada da matéria orgânica e a diminuição da quantidade e diversidade de organismos do solo (Moura, 2004; Leite et al., 2010).

Neste sentido, diversos estudos têm sido realizados com o intuito de se identificar sistemas de manejo que promovam aumento da qualidade do solo (Salmi et al., 2009), como os Sistemas Agroflorestais (SAF's), caracaterizados principalmente pela combinação de espécies florestais com cultivos agrícolas e adicionados ou não às atividades pecuárias (Lima et al., 2010). A presença de componentes florestais arbóreos nos SAF's adicionados a uma grande biodiversidade de espécies, propicia a deposição contínua de resíduos vegetais, o que facilita a manutenção da matéria orgânica do solo(Oelbermann et al., 2006; Smiley \& Kruschel, 2008) afetando diretamente os atributos físicos (Saha et al., 2001), químicos e biológicos do solo (Delabie et al., 2007; Huerta et al., 2007; Norgrove et al., 2009). Em última análise, o SAF proporciona benefícios ambientais, como a conservação da biodiversidade, o sequestro de carbono e a melhoria no controle de qualidade da água (Reitsma et al., 2001; McNeely \& Schroth, 2006; Nair, 2008).

Para avaliação da viabilidade ambiental de um agrossistema, convencional ou conservacionista tem-se sugerido como ferramenta, o monitoramento da qualidade do solo (D'Andrea et al., 2002; Sharma et al., 2005). Em se tratando da qualidade química, são muitos os estudos que demonstram modificações desta propriedade no solo em função dos diferentes manejos dos sistemas agrícolas (Muzilli, 1983; Silva \& Silveira, 2002; Wastowski et al., 2010). Ademais e ante as alterações nos teores de nutrientes do solo e se considerando que o conhecimento da fertilidade dos solos é um dos fatores primordiais para a obtenção de sucesso na atividade agrícola, diversos autores têm quantificado os atributos químicos do solo, sob diferentes usos e manejos (Perin et al., 2003; Lopes et al., 2006; Mota et al., 2007; Frazão et al., 2008) com o intuito de identificar aqueles considerados sustentáveis em médio e longo prazos.

Diferentemente do bioma Amazônia, em que há inúmeros trabalhos com SAF's, (Schroth et al., 2002; Barreto \& Lima, 2006; Maia et al., 2006) são escassos, no bioma Cerrado da Região Nordeste, trabalhos que visem associar esses sistemas conservacionistas à qualidade do solo (Lima et al., 2011). Desta forma, a meta deste trabalho foi quantificar os efeitos da adoção de SAF's sobre os atributos químicos de um Argissolo Vermelho-Amarelo em áreas de cerrado no estado do Piauí, em diferentes períodos do ano.

\section{MATERIAL E MÉTODOS}

O estudo foi realizado no município de Esperantina ( $03^{\circ} 54^{\prime}$ 07' S e 42 14 ' 02 "' W, altitude 59 m), localizado na mesorregião do Norte do Estado do Piauí, na microrregião do Baixo Parnaíba sob domínio do Cerrado, região Nordeste do Brasil. A precipitação pluvial média anual é de $1.500 \mathrm{~mm}$ e as temperaturas médias anuais variam de 26 a $34^{\circ} \mathrm{C}$. O solo das áreas em estudo é classificado como Argissolo Vermelho-Amarelo (Santos, 2006).

A região é caracterizada por apresentar uma formação vegetal predominante de transição entre os biomas Cerrado e floresta secundária mista (PLANAP, 2006), possuindo também extensas áreas com babaçuais, vegetação que caracteriza a chamada região Meio-Norte.

As áreas de estudo foram selecionadas em virtude da adoção dos sistemas agroflorestais, como sistema de manejo na produção agrícola familiar. O início do cultivo ocorreu por volta da década de 40, após a remoção da floresta nativa do cerrado e subsequente cultivo de culturas anuais, sobretudo milho e feijão, calcados na prática de corte e queima. Em 1997, com uma experiência piloto em uma das áreas, o manejo foi modificado, esse implantou o sistema agroflorestal (SAF); sequencialmente, em outras áreas da região também foram implantados os SAF's.

Foram selecionados nessas áreas, para estudo, os seguintes sistemas: Agroflorestal com seis anos de adoção (SAF6); agroflorestal com treze anos (SAF13); Agricultura de corte e queima (ACQ) com seis anos de cultivo contínuo com monoculturas de ciclo anual e floresta nativa de cerrado (FN) (Tabela 1). No SAF6 a prática do corte e a queima da vegetação foram utilizadas até 2003; a partir de 2004, após a regeneração natural da vegetação, o manejo foi realizado apenas com a roçada no final da estação seca, deixando o material vegetal sobre o solo. A segunda roçada foi realizada no período chuvoso, visando garantir o crescimento das espécies agrícolas. Desta forma, o cultivo vem sendo realizado, completando seis anos de adoção.

$\mathrm{Na}$ área com SAF13, o manejo convencional utilizando o corte e queima foi substituído em 1997, por um manejo com base ecológica. O material vegetal decorrente da roçada antes queimado passou a servir como cobertura do solo. A partir de então e ao longo do tempo, foram introduzidas espécies frutíferas e se permitiu, paralelamente, a regeneração natural de plantas pioneiras nas quais foram realizadas podas periódicas. Além disso, foram adicionados, ao solo, esterco de caprinos e todos os resíduos orgânicos decorrentes do consumo da família. Nas áreas sob os dois SAF's, as espécies arbóreas e frutíferas são distribuídas de forma aleatória, com maior densidade da espécie caju, cerca de quarenta por hectare, mamona, banana e manga em menor proporção. Aárea de ACQ é mantida sob queimas constantes com intervalos de um ano de pousio; ressalta-se que a última queima na área foi realizada em agosto de 2009 e nesta área são cultivados arroz e milho.

As coletas de solo foram realizadas em dois períodos distintos, em outubro de 2009, considerado período seco da região, e em março de 2010, período chuvoso (Figura 1). Em cada sistema foram abertas cinco minitrincheiras e coletadas quatro amostras simples para formar uma amostra composta, 
Tabela 1. Descrição das principais espécies presentes em cada sistema de manejo*
Sistemas Culturas/Espécies presentes
Milho (Zea may L.), abóbora (Curcubita pepo L.), fava (Phaseolus lunatus L.), mandioca (Manihot esculeta Crantz.), batata-doce (Ipomoea batatas Lam.) e algodão (Goossypium herbaceum L.) associadas à acerola (Malpighia glabra L.), mamão (Carica papaya L.), goiaba (Psidium guajava L.), banana (Musa paradisiaca L.), caju (Anacardium occidentale L.), manga (Mangifera indica L.), pinha
SAF 13 anos (Annona squamosa L.), pitomba (Talisia esculenta Raldlk.), mamona (Ricinus communis L), urucum (Bixa orellana L.), gergelim (Sesamum indicum L.), pau-d'arco (Tabebuia sp.), babaçu (Attalea speciosa Mart. ex Spreng.), gonçalo-alves (Astromiun fraxinifolium Schott), tamboril (Enterolobium sp.), jatobá (Hymenaea sp.), aroeira (Myracrodruon urundeuva Allemão), mufumbo (Combretum sp.) e unha-de-gato (Mimosa sp.).
SAF 6 Milho (Zea may L.) e mandioca (Manihot esculeta Crantz.) associadas às espécies como mamão (Carica papaya L.), caju (Anacardium occidentale L.), manga (Mangifera indica L.), melancia (Citrullus vulgaris Schrad.), maxixe (Cucumis anguria L.) e banana (Musa paradisiaca L.), mufumbo (Combretum sp.) e unha-de-gato (Mimosa sp.).
ACQ Arroz (Oryza sativa) e milho (Zea may L.).
FN Vegetação de floresta semidecídua preservada, na qual são observadas espécies de cerrado e caatinga, predominantemente Cerrado.

* (FN - Floresta nativa de cerrado; SAF13 - Sistema agroflorestal com 13 anos de adoção; SAF 6 - Sistema agroflorestal com 6 anos de adoção e ACQ - Agricultura de corte e queima Fonte: Dados obtidos in loco por meio de entrevista com os proprietários das áreas e complementados segundo Lima (2008)

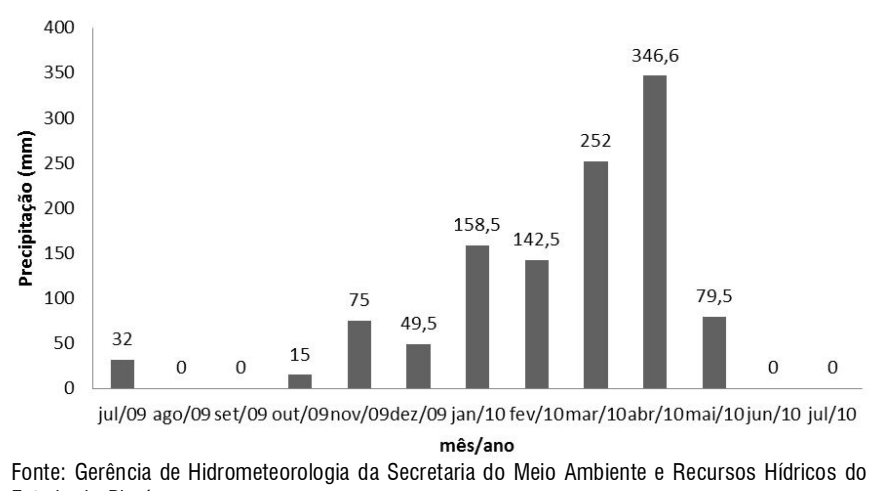

Fonte: Gerência
Estado do Piauí

Figura 1. Precipitação pluvial referente ao segundo semestre de 2009 e primeiro semestre de 2010, no município de Esperantina, PI

nas profundidades 0-5, 5-10, 10-20 e 20-40 cm. As amostras foram acondicionadas em sacos plásticos identificados e transportados ao Laboratório de Solos da Embrapa Meio Norte, Teresina, estado do Piauí.

Em laboratório, as amostras foram destorroadas, secadas e passadas em peneiras com malha de $2 \mathrm{~mm}$ para avaliação das características químicas do solo. Determinaram-se o $\mathrm{pH}$ do solo em água $(1: 2,5)$ por potenciometria, a acidez trocável $\left(\mathrm{Al}^{+3}\right)$ extraída com $\mathrm{KCl} 1 \mathrm{~mol} \mathrm{~L}^{-1}$ e quantificada por titulometria com hidróxido de sódio $0,025 \mathrm{~mol} \mathrm{~L}^{-1}$ padronizado com biftalato de potássio (EMBRAPA, 1997). O fósforo e o potássio foram extraídos com Mehlich 1 e determinados por colorimetria e fotometria de chama, respectivamente. A determinação do cálcio e o magnésio foi por espectrofotometria de absorção atômica, extraídos com $\mathrm{KCl} \mathrm{mol} \mathrm{L}^{-1}$. O Nitrogênio total (NT) do solo foi determinado por meio da digestão sulfúrica e dosado por destilação Kjedhal (Bremner et al., 1982). Para determinação dos teores de carbono orgânico total (COT), as amostras de solo foram trituradas em almofariz e passadas em peneira de malha $0,2 \mathrm{~mm}$, quantificadas por oxidação de matéria orgânica via úmida, utilizando-se solução de dicromato de potássio em meio ácido, com fonte externa de calor (Yeomans \& Bremner, 1988).

Os dados foram submetidos à análise de variância, sendo as médias dentro de cada profundidade nos diferentes sistemas de manejo comparadas pelo teste de Tukey em nível de 5\% de probabilidade.

\section{RESULTADOS E DISCUSSÃO}

$\mathrm{O}$ pH do solo foi superior nas áreas sob os SAF's em todas as profundidades em relação ao solo sob FN e ACQ (Tabela 2). Logo, a adoção do SAF em relação à ACQ em um Argissolo Vermelho-Amarelo, que possui caráter ácido, segundo sua classificação (EMBRAPA, 2006), promoveu aumento do $\mathrm{pH}$ principalmente no solo sob o SAF com maior tempo de adoção (SAF13). Este fato pode estar relacionado com a maior disponibilidade da matéria orgânica do solo (MOS).

A MOS possui a função de complexar os cátions $\mathrm{H}^{+}$e $\mathrm{Al}^{+3}$ livres com compostos orgânicos aniônicos dos resíduos e adicionar bases ( $\mathrm{Ca}, \mathrm{Mg}$ e $\mathrm{K}$ ) que reduzem a acidez do solo e aumentam o pH (Franchini et al., 2001; Pavinato, 2008). A ação da MOS sobre o pH em relação à área que possui a prática do corte e queima (Tabela 2), não foi eficiente visto que se tem, nesta área, grandes perdas de MOS, pelo processo da queima. Nessas áreas os valores de $\mathrm{pH}$ foram muito baixos, sobremaneira nas profundidades de $10-20 \mathrm{~cm}(4,22)$ e $20-40 \mathrm{~cm}$ $(4,15)$. Em nenhum dos sistemas foi verificado o efeito da sazonalidade sobre o $\mathrm{pH}$.

Inversamente aos valores de $\mathrm{pH}$, os teores de $\mathrm{Al}^{+3}$ foram menores no SAF13, em especial nas camadas superficiais, seguido do SAF6 e FN e os maiores teores ocorreram no solo sob ACQ (Tabela 2). Nos SAF's e FN, a diminuição dos teores de $\mathrm{Al}^{+3}$ tem relação com efeito da MOS, complexando o alumínio livre em solução. No que se refere ao solo sob ACQ, a diminuição na camada superficial do teor de cátions livres $\mathrm{H}^{+}$e $\mathrm{Al}^{+3}$ pode estar relacionada com a influência das cinzas sobre as características químicas do solo que atua neutralizando a acidez do solo pela ação de componentes básicos ( $\mathrm{Ca}, \mathrm{Mg}$ e $\mathrm{K})$ que são liberados após a queima da vegetação (Silva \& Silva, 2006). Ressalta-se, porém, que embora a queima dos resíduos vegetais possa elevar os teores das bases trocáveis e diminuir os teores de $\mathrm{H}^{+}$e $\mathrm{Al}^{+3}$, ela poderá, também, a longo prazo, diminuir a fertilidade do solo, uma vez que as cinzas são facilmente carreadas por lixiviação e/ou erosão (Mendonza et al., 2000). 
Tabela 2. Valores de $\mathrm{pH}$, acidez trocável $\left(\mathrm{Al}^{+3}\right)$, acidez potencial $(\mathrm{H}+\mathrm{Al})$ e saturação por alumínio $(\mathrm{m})$ em um Argissolo Vermelho Amarelo sob sistemas agroflorestais, no cerrado do estado do Piauí

\begin{tabular}{|c|c|c|c|c|c|c|c|c|}
\hline \multirow{3}{*}{ Sistemas } & \multicolumn{2}{|c|}{$\mathrm{pH}\left(\mathrm{H}_{2} \mathrm{O}\right)$} & \multicolumn{2}{|c|}{$\mathrm{Al}^{+3}$} & \multicolumn{2}{|c|}{$\mathrm{H}+\mathrm{Al}$} & \multicolumn{2}{|c|}{$\mathbf{m} \%$} \\
\hline & \multicolumn{8}{|c|}{$\mathrm{cmol}_{\mathrm{c}} \mathrm{dm}^{-3}$} \\
\hline & Seco & Chuvoso & Seco & Chuvoso & Seco & Chuvoso & Seco & Chuvoso \\
\hline & \multicolumn{8}{|c|}{$0-5 \mathrm{~cm}$} \\
\hline FN & $5,02 \mathrm{bc}$ & $4,89 \mathrm{c}$ & $0,74 \mathrm{~b}$ & $0,68 \mathrm{~b}$ & $5,36 \mathrm{a}$ & $5,28 \mathrm{a}$ & $9,41 \mathrm{~b}$ & $24,66 \mathrm{~b}$ \\
\hline SAF13 & $6,10 \mathrm{a}$ & $6,10 \mathrm{a}$ & $0,30 \mathrm{c}$ & $0,32 \mathrm{c}$ & $3,24 \mathrm{c}$ & $3,03 \mathrm{c}$ & $1,38 \mathrm{c}$ & $6,52 \mathrm{c}$ \\
\hline SAF6 & $5,84 \mathrm{a}$ & $5,60 \mathrm{~b}$ & $0,20 \mathrm{c}$ & $0,16 \mathrm{c}$ & $4,38 \mathrm{bc}$ & $4,42 \mathrm{bc}$ & $2,21 \mathrm{C}$ & $5,23 \mathrm{c}$ \\
\hline \multirow[t]{2}{*}{$A C Q$} & $4,80 \mathrm{C}$ & $5,04 \mathrm{c}$ & $1,26 \mathrm{a}$ & $1,70 \mathrm{a}$ & $4,79 a b$ & $4,72 \mathrm{ab}$ & $13,50 \mathrm{a}$ & $69,62 \mathrm{a}$ \\
\hline & \multicolumn{8}{|c|}{$5-10 \mathrm{~cm}$} \\
\hline $\mathrm{FN}$ & $5,04 \mathrm{~b}$ & $4,70 \mathrm{c}$ & $1,00 \mathrm{~b}$ & $0,94 \mathrm{~b}$ & $5,51 \mathrm{a}$ & $5,25 \mathrm{a}$ & $21,22 \mathrm{~b}$ & $45,73 \mathrm{~b}$ \\
\hline SAF13 & $6,01 \mathrm{a}$ & $6,13 a b$ & $0,18 \mathrm{c}$ & $0,18 \mathrm{c}$ & $2,66 \mathrm{c}$ & $2,67 d$ & $1,07 \mathrm{~d}$ & $4,98 d$ \\
\hline SAF6 & $5,67 a b$ & $5,72 \mathrm{~b}$ & $0,20 \mathrm{c}$ & $0,16 \mathrm{c}$ & $4,68 a b$ & $4,65 a b$ & $2,85 \mathrm{~cd}$ & $7,24 \mathrm{~cd}$ \\
\hline \multirow[t]{2}{*}{$\mathrm{ACQ}$} & $4,28 \mathrm{c}$ & $4,61 \mathrm{c}$ & $1,70 \mathrm{a}$ & $1,86 \mathrm{a}$ & $3,87 \mathrm{~b}$ & $3,50 \mathrm{~cd}$ & $52,62 \mathrm{a}$ & $80,08 \mathrm{a}$ \\
\hline & \multicolumn{8}{|c|}{$10-20 \mathrm{~cm}$} \\
\hline FN & $4,84 \mathrm{c}$ & $4,72 \mathrm{c}$ & $1,48 b$ & $1,34 \mathrm{~b}$ & $5,18 \mathrm{a}$ & $4,98 \mathrm{a}$ & $36,65 \mathrm{~b}$ & $64,16 \mathrm{~b}$ \\
\hline SAF13 & $5,85 a b$ & $6,21 \mathrm{a}$ & $0,10 \mathrm{~d}$ & $0,14 d$ & $3,21 \mathrm{c}$ & $3,03 d$ & $0,90 \mathrm{~d}$ & $2,76 \mathrm{~d}$ \\
\hline SAF6 & $5,32 b$ & $5,52 \mathrm{~b}$ & $0,40 \mathrm{~cd}$ & $0,34 \mathrm{~cd}$ & $4,39 \mathrm{~b}$ & $4,42 \mathrm{ab}$ & $9,96 \mathrm{C}$ & $21,07 \mathrm{C}$ \\
\hline \multirow[t]{2}{*}{$A C Q$} & $4,22 \mathrm{c}$ & $4,54 \mathrm{c}$ & $1,80 \mathrm{a}$ & $1,80 a b$ & $3,95 \mathrm{bc}$ & $3,27 \mathrm{~cd}$ & $69,38 \mathrm{a}$ & $84,91 \mathrm{a}$ \\
\hline & \multicolumn{8}{|c|}{$20-40 \mathrm{~cm}$} \\
\hline FN & $4,85 \mathrm{bc}$ & $4,77 \mathrm{c}$ & $1,30 \mathrm{ab}$ & $1,40 \mathrm{a}$ & $5,39 a$ & $5,28 \mathrm{a}$ & $34,09 \mathrm{c}$ & $57,17 \mathrm{~b}$ \\
\hline SAF13 & $5,75 \mathrm{a}$ & $6,06 \mathrm{a}$ & $0,12 \mathrm{~d}$ & $0,14 d$ & $3,19 c$ & $3,10 \mathrm{c}$ & $1,52 \mathrm{~d}$ & $3,88 \mathrm{~d}$ \\
\hline SAF6 & $5,40 a b$ & $5,34 \mathrm{~b}$ & $0,56 \mathrm{c}$ & $0,48 \mathrm{~cd}$ & $3,89 \mathrm{bc}$ & $3,60 \mathrm{bc}$ & $57,81 \mathrm{~b}$ & $29,99 \mathrm{c}$ \\
\hline$A C Q$ & $4,15 \mathrm{c}$ & $4,51 \mathrm{c}$ & $1,54 \mathrm{a}$ & $1,70 a b$ & $4,16 \mathrm{~b}$ & $3,66 \mathrm{bc}$ & $74,08 \mathrm{a}$ & $85,82 \mathrm{a}$ \\
\hline
\end{tabular}

Médias seguidas da mesma letra nas colunas não diferem entre si pelo teste de Tukey em nível de $5 \%$ de significância. FN - floresta nativa de cerrado; SAF6: sistema agroflorestal com seis anos de adoção; SAF13 - sistema agroflorestal com treze anos de adoção e ACQ - Agricultura de corte e queima

Entre os dois períodos coletados não houve diferenciação quanto aos teores de $\mathrm{Al}^{+3}$ para nenhum dos sistemas estudados. Em todas as profundidades se observaram maiores teores de $\mathrm{H}+\mathrm{Al}$ no solo sob $\mathrm{FN}$ e os menores no SAF13 (Tabela 2) o que também pode ser explicado pelos maiores teores de MOS em referência ao solo sob FN e ACQ (Tabela 2).
Os teores de cálcio, magnésio e potássio foram, em todas as profundidades, mais elevados $(\mathrm{p}<0,05)$ nos solos sob os SAF's, superando os teores verificados na FN (Tabela 3). Os altos teores de nutrientes (as bases trocáveis $\mathrm{Ca}, \mathrm{Mg}$ e $\mathrm{K}$ ) verificados nos solos sob os SAF's (Tabela 3) podem estar associados à alta atividade de decomposição realizada pela biota do solo que este ambiente proporciona (Lima, 2008). Além

Tabela 3. Teores de potássio (K), cálcio (Ca) e magnésio (Mg) em um Argissolo Vermelho Amarelo sob diferentes Sistemas Agroflorestais no Cerrado do Estado do Piauí

\begin{tabular}{|c|c|c|c|c|c|c|}
\hline \multirow{3}{*}{ Sistemas } & \multicolumn{2}{|c|}{$\mathrm{K}^{+}$} & \multicolumn{2}{|c|}{$\mathrm{Ca}^{2+}$} & \multicolumn{2}{|c|}{$\mathrm{Mg}^{2+}$} \\
\hline & \multicolumn{6}{|c|}{$\mathrm{cmol}_{\mathrm{c}} \mathrm{dm}^{-3}$} \\
\hline & Seco & Chuvoso & Seco & Chuvoso & Seco & Chuvoso \\
\hline $\begin{array}{l}\text { FN } \\
\text { SAF13 } \\
\text { SAF6 } \\
\text { ACQ }\end{array}$ & $\begin{array}{l}0,32 \mathrm{~b} \\
0,46 \mathrm{a} \\
0,38 \mathrm{~b} \\
0,34 \mathrm{~b}\end{array}$ & $\begin{array}{l}0,33 \mathrm{a} \\
0,34 \mathrm{a} \\
0,38 \mathrm{a} \\
0,15 \mathrm{~b}\end{array}$ & $\begin{array}{r}4,82 \mathrm{c} \\
18,24 \mathrm{a} \\
7,11 \mathrm{~b} \\
4,49 \mathrm{c} \\
\end{array}$ & $\begin{array}{l}1,46 \mathrm{c} \\
3,42 \mathrm{a} \\
2,39 \mathrm{~b} \\
0,29 \mathrm{~d} \\
\end{array}$ & $\begin{array}{l}1,99 \mathrm{bc} \\
3,18 \mathrm{a} \\
1,55 \mathrm{c} \\
3,27 \mathrm{a} \\
\end{array}$ & $\begin{array}{l}0,33 \mathrm{bc} \\
0,84 \mathrm{a} \\
0,23 \mathrm{c} \\
0,29 \mathrm{c}\end{array}$ \\
\hline $\begin{array}{l}\text { FN } \\
\text { SAF13 } \\
\text { SAF6 } \\
\text { ACQ }\end{array}$ & $\begin{array}{l}0,26 a b \\
0,32 a \\
0,22 b \\
0,24 a b \\
\end{array}$ & $\begin{array}{l}0,26 \mathrm{a} \\
0,29 \mathrm{a} \\
0,29 \mathrm{a} \\
0,12 \mathrm{~b} \\
\end{array}$ & $\begin{array}{r}2,07 \mathrm{c} \\
13,50 \mathrm{a} \\
5,92 \mathrm{~b} \\
0,85 \mathrm{~d} \\
\end{array}$ & $\begin{array}{l}0,63 \mathrm{c} \\
2,59 \mathrm{a} \\
1,66 \mathrm{~b} \\
0,13 \mathrm{~d}\end{array}$ & $\begin{array}{l}1,40 \mathrm{~b} \\
2,39 \mathrm{a} \\
0,87 \mathrm{c} \\
0,57 \mathrm{c}\end{array}$ & $\begin{array}{l}0,21 \mathrm{~b} \\
0,50 \mathrm{a} \\
0,14 \mathrm{c} \\
0,20 \mathrm{~b}\end{array}$ \\
\hline $\begin{array}{l}\text { FN } \\
\text { SAF13 } \\
\text { SAF6 } \\
\text { ACQ } \\
\end{array}$ & $\begin{array}{l}0,23 a b \\
0,20 b \\
0,23 a b \\
0,13 c\end{array}$ & $\begin{array}{l}0,21 \mathrm{~b} \\
0,29 \mathrm{a} \\
0,26 \mathrm{ab} \\
0,07 \mathrm{c} \\
\end{array}$ & $\begin{array}{l}1,37 \mathrm{c} \\
9,79 \mathrm{a} \\
3,09 \mathrm{~b} \\
0,20 \mathrm{c}\end{array}$ & $\begin{array}{l}0,29 \mathrm{bc} \\
4,21 \mathrm{a} \\
0,87 \mathrm{~b} \\
0,14 \mathrm{c} \\
\end{array}$ & $\begin{array}{l}1,01 \mathrm{a} \\
1,32 \mathrm{a} \\
0,44 \mathrm{~b} \\
0,46 \mathrm{~b}\end{array}$ & $\begin{array}{l}0,22 \mathrm{~b} \\
0,42 \mathrm{a} \\
0,12 \mathrm{~cd} \\
0,10 \mathrm{~d}\end{array}$ \\
\hline $\begin{array}{l}\text { FN } \\
\text { SAF13 } \\
\text { SAF6 } \\
\text { ACQ }\end{array}$ & $\begin{array}{l}0,18 \mathrm{~b} \\
0,18 \mathrm{~b} \\
0,21 \mathrm{a} \\
0,11 \mathrm{c}\end{array}$ & $\begin{array}{l}0,30 \mathrm{a} \\
0,34 \mathrm{a} \\
0,29 \mathrm{a} \\
0,08 \mathrm{~b}\end{array}$ & $\begin{array}{l}1,16 \mathrm{~b} \\
6,71 \mathrm{a} \\
0,12 \mathrm{c} \\
0,13 \mathrm{c}\end{array}$ & $\begin{array}{l}0,34 \mathrm{~b} \\
2,81 \mathrm{a} \\
0,67 \mathrm{~b} \\
0,13 \mathrm{c}\end{array}$ & $\begin{array}{l}0,95 \mathrm{a} \\
0,93 \mathrm{a} \\
0,05 \mathrm{c} \\
0,31 \mathrm{~b}\end{array}$ & $\begin{array}{l}0,18 \mathrm{bc} \\
0,33 \mathrm{a} \\
0,14 \mathrm{c} \\
0,06 \mathrm{~d}\end{array}$ \\
\hline
\end{tabular}

Médias seguidas da mesma letra nas colunas não diferem entre si pelo teste de Tukey em nível de 5\% de significância. FN - Floresta nativa de cerrado; SAF6 - Sistema agroflorestal com seis anos de adoção; SAF13 - Sistema agroflorestal com treze anos de adoção e ACQ - Agricultura de corte e queima 
disso, a presença do componente arbóreo nesses sistemas promove maior deposição de material vegetal (raízes, galhos, folhas) que é mineralizado, influenciando as reações químicas no solo e contribuindo para a melhoria dos parâmetros de fertilidade do solo sob SAF (Campanha et al., 2007).

Em relação à $\mathrm{FN}$, os teores de $\mathrm{Ca}, \mathrm{Mg}$ e $\mathrm{K}$ foram inferiores (Tabela 3) comparados aos SAF's, uma vez que na FN, um ambiente em equilíbrio, o que está disponível no solo é provável que esteja sendo absorvido pela vegetação sem promover maior disponibilização desses nutrientes para a solução do solo (Barreto \& Lima, 2006). Este fato também pode estar relacionado com a presença de culturas anuais que melhor expõem as frações orgânicas aos micro-organismos decompositores e também que proporcionam maior quebra de macroagregados, pelo revolvimento do solo (Silva et al., 1999; Barreto \& Lima, 2006).

Durante o período chuvoso observou-se perda dos nutrientes ( $\mathrm{Ca}, \mathrm{Mg}$ e $\mathrm{K}$ ) passível de ter sido causada pela enxurrada e demais ações da chuva, fato bastante evidenciado pela literatura, mesmo em sistemas de maior estabilização (Tabela 3), como os SAF's e as áreas de mata nativa, evento que pode ser corroborado por Lobato (2009) que constatou consideráveis perdas de nutrientes em um Argissolo Vermelho Amarelo em áreas sob SAF no Estado do Ceará. Nos SAF's, por se aproximarem mais dos sistemas naturais na ocorrência de estresse causado ou não por diferenças climáticas, a resiliência do sistema permitirá o restabelecimento da capacidade de suporte dos nutrientes aos níveis aceitáveis, semelhantes às áreas de floresta nativa (Dias, 2002).

A CTC T e CTC $t$ foram superiores nos SAF's. A eficiência nas reações de troca de cargas nos sistemas sob SAF's pode estar relacionada à presença das raízes das árvores que atuam na melhoria da ciclagem dos nutrientes (Tabela 4). Observou- se então, nas profundidades $0-5$ e $5-10 \mathrm{~cm}$, que os valores de CTC T foram similares nos solos sob FN e sob os SAF's, superiores portanto em relação a ACQ. Além disso, a CTC $\mathrm{t}$ foi superior em todas as profundidades no SAF13, seguidas do SAF6 e FN (Tabela 4).

Ademais, observou-se diminuição da CTC T e da CTC t ao longo do perfil, em todos os sistemas e no período chuvoso em relação ao período seco. Com melhor distribuição dos nutrientes nos SAF's ao longo do perfil associada à atuação conjunta do bombeamento de nutrientes pelas raízes das árvores das camadas mais profundas do solo, para a superfície, potencializado pelo manejo do sistema, ocorre dinamização da atuação da biota do solo para disponibilização de nutrientes, em função do incremento periódico de matéria orgânica de qualidade diferenciada, resultante do manejo (Peneireiro, 1999). A troca de cátions de solos com maiores teores de MOS, tal como os SAF's, também é influenciada pelo alto poder de reatividade da MOS, diretamente relacionada aos seus diversificados radicais orgânicos que a mesma possui (Tabela 4).

No SAF13, seguido do SAF6 também ocorreram os maiores valores de saturação por base (V\%), intermediários na FN e menores no solo sob ACQ. Além do sistema de manejo a profundidade exerceu efeito significativo sobre o V\%, diminuindo com o seu aumento sendo que no período chuvoso também ocorreram diminuições do V\%.

Em todas as profundidades os teores de COT foram superiores na área sob o SAF13, intermediários no SAF6 e FN e inferiores no solo sob ACQ, exceto na profundidade $0-5 \mathrm{~cm}$ do período seco, quando então o teor de $\mathrm{C}$ do solo sob ACQ não apresentou diferença significativa em relação ao SAF13 (Tabela 5). Com o aumento da profundidade, ocorreu diminuição dos teores de $\mathrm{C}$ do solo, nas quatro áreas.

Tabela 4. Capacidade de troca de cátions (CTC) potencial (T) e efetiva (t) e saturação por base (V\%) em um Argissolo Vermelho Amarelo sob sistemas agroflorestais no cerrado do estado do Piauí

\begin{tabular}{|c|c|c|c|c|c|c|}
\hline \multirow{3}{*}{ Sistemas } & \multicolumn{2}{|c|}{ CTC T } & \multicolumn{2}{|c|}{ CTC t } & \multicolumn{2}{|c|}{ V\% } \\
\hline & \multicolumn{6}{|c|}{$\mathrm{cmol}_{\mathrm{c}} \mathrm{dm}^{-3}$} \\
\hline & Seco & Chuvoso & Seco & Chuvoso & Seco & Chuvoso \\
\hline FN & $12,50 \mathrm{c}$ & $7,40 \mathrm{a}$ & $7,88 \mathrm{c}$ & $2,80 \mathrm{bc}$ & $57,17 \mathrm{c}$ & $28,62 \mathrm{c}$ \\
\hline SAF13 & $25,14 \mathrm{a}$ & $7,64 \mathrm{a}$ & $22,19 \mathrm{a}$ & $4,93 \mathrm{a}$ & $86,89 \mathrm{a}$ & $60,41 \mathrm{a}$ \\
\hline SAF6 & $13,43 \mathrm{~b}$ & $7,43 \mathrm{a}$ & $9,25 \mathrm{~b}$ & $3,17 \mathrm{~b}$ & $66,96 \mathrm{~b}$ & $40,31 b$ \\
\hline$A C Q$ & \multicolumn{6}{|c|}{$5-10 \mathrm{~cm}$} \\
\hline FN & $9,25 \mathrm{c}$ & $6,36 \mathrm{a}$ & $4,74 \mathrm{~cd}$ & $2,05 \mathrm{C}$ & $40,34 \mathrm{c}$ & $17,48 \mathrm{~cd}$ \\
\hline SAF13 & $18,89 \mathrm{a}$ & $6,06 \mathrm{a}$ & $16,40 \mathrm{a}$ & $3,57 \mathrm{a}$ & $85,79 \mathrm{a}$ & $56,28 \mathrm{a}$ \\
\hline SAF6 & $11,70 \mathrm{~b}$ & $6,75 \mathrm{a}$ & 7,22 b & $2,26 \mathrm{bc}$ & $59,56 \mathrm{~b}$ & $30,81 \mathrm{~b}$ \\
\hline \multirow[t]{2}{*}{$A C Q$} & $5,54 d$ & $3,96 \mathrm{~b}$ & $3,37 d$ & $2,32 \mathrm{~b}$ & $29,01 d$ & $12,09 d$ \\
\hline & \multicolumn{6}{|c|}{$10-20 \mathrm{~cm}$} \\
\hline FN & $7,80 \mathrm{C}$ & $5,71 \mathrm{~b}$ & $4,10 b$ & $2,06 \mathrm{~b}$ & $33,32 \mathrm{c}$ & $12,83 \mathrm{~cd}$ \\
\hline SAF6 & 8,16 bc & $5,69 \mathrm{~b}$ & 4,16b & $1,61 \mathrm{c}$ & $45,38 \mathrm{~b}$ & $22,32 \mathrm{~b}$ \\
\hline \multirow[t]{2}{*}{$A C Q$} & $4,75 \mathrm{~d}$ & $3,59 \mathrm{c}$ & $2,60 \mathrm{c}$ & $2,12 b$ & $16,76 \mathrm{~d}$ & $9,63 \mathrm{~d}$ \\
\hline & \multicolumn{6}{|c|}{$20-40 \mathrm{~cm}$} \\
\hline FN & $4,28 \mathrm{C}$ & $6,11 b$ & $3,60 \mathrm{~b}$ & $2,23 \mathrm{~b}$ & 29,82 b & $13,79 \mathrm{c}$ \\
\hline SAF13 & $6,16 b$ & $6,59 a b$ & $7,95 \mathrm{a}$ & $3,63 \mathrm{a}$ & $69,77 \mathrm{a}$ & $53,09 a$ \\
\hline SAF6 & $4,72 \mathrm{c}$ & $4,70 \mathrm{c}$ & $0,95 d$ & $1,58 d$ & $9,18 d$ & $23,69 \mathrm{~b}$ \\
\hline$A C Q$ & $11,02 \mathrm{a}$ & $3,94 \mathrm{~d}$ & $2,10 \mathrm{C}$ & $1,98 \mathrm{~cd}$ & $11,57 \mathrm{~cd}$ & $7,130 \mathrm{~d}$ \\
\hline
\end{tabular}

Médias seguidas da mesma letra nas colunas não diferem entre si pelo teste de Tukey em nível de 5\% de significância. FN - Floresta nativa de cerrado; SAF6 - Sistema agroflorestal com seis anos de adoção; SAF13 - Sistema agroflorestal com treze anos de adoção e ACQ - Agricultura de corte e queima 
Tabela 5. Teores totais de carbono orgânico (COT) e nitrogênio (NT), relação carbono e nitrogênio (C/N) e teor de fósforo (P) disponível em Argissolo Vermelho Amarelo sob Sistemas Agroflorestais no Cerrado do Estado do Piauí

\begin{tabular}{|c|c|c|c|c|c|c|c|c|}
\hline \multirow{3}{*}{ Sistemas } & \multicolumn{2}{|c|}{$\bar{C}$} & \multicolumn{2}{|c|}{$\mathbf{N}$} & \multicolumn{2}{|c|}{$\mathrm{C} / \mathrm{N}$} & \multirow{2}{*}{\multicolumn{2}{|c|}{$\begin{array}{c}\mathrm{P} \\
\mathrm{mg} \mathrm{dm^{-3 }}\end{array}$}} \\
\hline & \multicolumn{4}{|c|}{$\mathrm{g} \mathrm{kg}^{-1}$} & & & & \\
\hline & Seco & Chuvoso & Seco & Chuvoso & Seco & Chuvoso & Seco & Chuvoso \\
\hline & \multicolumn{8}{|c|}{$0-5 \mathrm{~cm}$} \\
\hline FN & $18,53 \mathrm{bc}$ & $21,56 \mathrm{a}$ & $1,55 b$ & $1,94 \mathrm{~b}$ & $12,04 \mathrm{a}$ & $11,50 a b$ & $6,11 \mathrm{a}$ & $2,10 \mathrm{~b}$ \\
\hline SAF13 & $23,86 \mathrm{a}$ & $22,91 \mathrm{a}$ & $2,25 \mathrm{a}$ & $2,72 \mathrm{a}$ & $10,94 \mathrm{~b}$ & 8,67 b & $1,91 \mathrm{~b}$ & $1,23 \mathrm{c}$ \\
\hline SAF6 & $15,74 \mathrm{c}$ & $19,35 b$ & $1,82 a b$ & $1,20 \mathrm{c}$ & $9,09 \mathrm{c}$ & $14,30 \mathrm{a}$ & $1,89 \mathrm{bc}$ & $2,40 \mathrm{~b}$ \\
\hline \multirow[t]{2}{*}{$A C Q$} & $19,75 b$ & $4,40 \mathrm{C}$ & $1,82 a b$ & $0,75 d$ & $10,76 b$ & $5,48 \mathrm{c}$ & $1,73 \mathrm{c}$ & $7,51 \mathrm{a}$ \\
\hline & \multicolumn{8}{|c|}{$5-10 \mathrm{~cm}$} \\
\hline FN & $10,01 \mathrm{~b}$ & $19,63 b$ & $0,97 \mathrm{c}$ & $1,59 \mathrm{bc}$ & $10,45 \mathrm{ab}$ & $13,22 \mathrm{a}$ & $5,99 \mathrm{~b}$ & $1,45 d$ \\
\hline SAF13 & $20,20 \mathrm{a}$ & $21,89 a b$ & $2,89 \mathrm{a}$ & $2,46 \mathrm{a}$ & $7,02 \mathrm{c}$ & $7,96 \mathrm{c}$ & $1,80 \mathrm{c}$ & $4,46 \mathrm{c}$ \\
\hline SAF6 & $11,58 \mathrm{~b}$ & $11,10 \mathrm{c}$ & $1,04 \mathrm{c}$ & $1,12 \mathrm{c}$ & $11,17 \mathrm{~b}$ & $9,72 \mathrm{~b}$ & $7,93 \mathrm{a}$ & $5,55 \mathrm{~b}$ \\
\hline \multirow[t]{2}{*}{ ACQ } & $7,94 \mathrm{c}$ & $4,28 d$ & $1,84 \mathrm{~b}$ & $0,54 \mathrm{~d}$ & $4,36 \mathrm{~d}$ & $8,90 \mathrm{bc}$ & $5,91 \mathrm{~b}$ & $7,63 \mathrm{a}$ \\
\hline & \multicolumn{8}{|c|}{$10-20 \mathrm{~cm}$} \\
\hline FN & $15,27 \mathrm{a}$ & $17,55 \mathrm{~b}$ & $1,49 b$ & $1,16 \mathrm{~b}$ & $10,23 a b$ & $15,34 \mathrm{a}$ & $2,35 \mathrm{c}$ & $1,78 \mathrm{c}$ \\
\hline SAF13 & $15,90 \mathrm{a}$ & $20,30 a$ & $2,30 \mathrm{a}$ & $1,41 \mathrm{ab}$ & $6,87 \mathrm{c}$ & $13,71 a b$ & $7,92 \mathrm{a}$ & $2,79 b$ \\
\hline SAF6 & $7,65 \mathrm{~b}$ & $8,75 \mathrm{c}$ & $0,76 \mathrm{~cd}$ & $0,76 \mathrm{~cd}$ & $10,18 a b$ & $11,78 \mathrm{~b}$ & $3,79 \mathrm{~b}$ & $2,32 \mathrm{~b}$ \\
\hline \multirow[t]{2}{*}{$A C Q$} & $5,28 \mathrm{c}$ & $2,32 \mathrm{~d}$ & $0,41 \mathrm{~d}$ & $0,36 \mathrm{~d}$ & $12,12 \mathrm{a}$ & $6,34 \mathrm{c}$ & $3,69 \mathrm{~b}$ & $6,29 \mathrm{a}$ \\
\hline & \multicolumn{8}{|c|}{$20-40 \mathrm{~cm}$} \\
\hline FN & $12,30 a b$ & $15,56 \mathrm{~b}$ & $1,37 \mathrm{~b}$ & $1,32 \mathrm{a}$ & $9,08 \mathrm{c}$ & $12,14 \mathrm{~b}$ & $1,75 \mathrm{c}$ & $1,57 \mathrm{~d}$ \\
\hline SAF13 & $11,16 \mathrm{~b}$ & $18,05 \mathrm{a}$ & $2,31 \mathrm{a}$ & $1,37 \mathrm{a}$ & $4,85 d$ & $12,82 \mathrm{a}$ & $5,82 \mathrm{a}$ & $3,79 \mathrm{~b}$ \\
\hline SAF6 & $8,14 \mathrm{C}$ & $8,12 \mathrm{C}$ & $0,58 \mathrm{~cd}$ & $0,62 \mathrm{~b}$ & $14,49 a b$ & $12,68 \mathrm{ab}$ & $1,67 \mathrm{C}$ & $2,49 \mathrm{c}$ \\
\hline$A C Q$ & $2,34 \mathrm{~d}$ & $0,63 \mathrm{~d}$ & $0,45 d$ & $0,26 \mathrm{c}$ & $13,97 \mathrm{~b}$ & $2,50 \mathrm{c}$ & $3,90 \mathrm{~b}$ & $4,38 \mathrm{a}$ \\
\hline
\end{tabular}

Médias seguidas da mesma letra nas colunas não diferem entre si pelo teste de Tukey em nível de $5 \%$ de significância. FN - Floresta nativa de cerrado; SAF6 - Sistema agroflorestal com seis anos de adoção; SAF13 - sistema agroflorestal com treze anos de adoção e ACQ - Agricultura de corte e queima

A ausência de revolvimento do solo nos SAF's e na mata nativa proporciona melhores condições para os organismos responsáveis pela fragmentação do material vegetal e ciclagem dos nutrientes (Cunha et al., 2012). Vários trabalhos têm evidenciado o potencial dos SAF's, sobretudo daqueles que utilizam o cultivo em aleias na ciclagem de nutrientes (Franzel et al., 2001), que atua no aumento dos teores de MOS e seus componentes, como C, Pe N (Tabela 5).

Assim, o SAF induz o aumento dos níveis de $\mathrm{C}$ do solo em virtude do maior aporte de resíduos que o sistema recebe ao longo dos anos (Barreto \& Lima, 2006) e com baixa interferência antrópica (Tabela 5). Referidos resultados condizem com Fonte et al. (2010) que também constataram a promoção do aumento dos teores de COT no solo sob SAF, em estudo realizado em Honduras. Contudo, no solo sob ACQ foram observados os menores teores em relação aos demais sistemas. Esses baixos teores observados na ACQ foram provocados principalmente pelas perdas de nutrientes quando da queima da vegetação durante o preparo do solo para o plantio (Sommer, 2000).

A diferenciação entre os teores de matéria orgânica do solo observada nos SAF's e ACQ (Tabela 5) está diretamente relacionada às mudanças nos sistemas de manejo que podem afetar os teores de carbono do solo pela alteração do aporte anual de resíduos vegetais e pela modificação na taxa de decomposição da MOS (matéria orgânica do solo) (Leite et al., 2003).

Quanto ao efeito da sazonalidade, constatou-se que os teores de $\mathrm{C}$ diminuíram no período chuvoso, fato devido, provavelmente, à deposição de folhedo e morte de raízes finas $(\varnothing<2 \mathrm{~mm})$ que ocorrem durante o período seco e que são as principais entradas de carbono no solo (Salcedo \& Sampaio, 2008). Com o início das chuvas a maior umidade no solo determina um forte aumento da atividade biológica com a consequente mineralização de parte do carbono aportado, diminuindo os teores do solo (Moreira, 2006).

Da mesma forma, os teores de $\mathrm{N}$ foram superiores nos SAF's, seguidos pela FN e inferiores no solo sob ACQ (Tabela 5). Maia et al. (2008) ressalvam que sistemas mais conservacionistas, como os SAF's, que priorizam o aporte de resíduos orgânicos e a menor mobilização do solo (Kaur et al., 2000) têm-se mostrado eficientes em manter e, às vezes, elevar, os teores de $\mathrm{N}$ (Tabela 5). Por outro lado, menores teores observados no solo sob ACQ, podem ser explicados também pelas perdas da matéria orgânica do solo, causadas pela prática convencional de corte e queima.

O manejo também exerceu efeito significativo sobre os teores de N no SAF6 e ACQ, os quais diminuíram com o aumento da profundidade, podendo este fato está relacionado à abundância de nutrientes nas camadas mais superficiais, com relação às camadas mais profundas, conforme Wastowski et al. (2010) que apontam maior concentração de nutrientes nas camadas superficiais em sistemas como SAF's em que há menor ou nenhuma movimentação de solo (Tabela 5). A sazonalidade exerceu efeito significativo apenas no solo sob ACQ, diminuindo no período chuvoso.

A relação $\mathrm{C} / \mathrm{N}$ foi superior nos SAF's e $\mathrm{FN}$ nas camadas superficiais e maior $\left(\mathrm{p}_{-}<0,05\right)$ no sistema ACQ, nas camadas de 10-20 e 20-40 cm, o que denota maior tempo do material orgânico sobre o solo, ou seja, maior relação C/N (Villatoro, 2004). Esta característica ocorre em SAF's principalmente pelo maior acúmulo e permanência de material orgânico sob o solo, em razão da estabilidade dos sistemas. No solo sob ACQ a relação $\mathrm{C} / \mathrm{N}$ superior em profundidades maiores $(10-20 \mathrm{e} 20-40 \mathrm{~cm})$ pode ser justificada pelo menor efeito da queima nessas profundidades, promovendo uma permanência maior do material orgânico do solo; logo, maior $\mathrm{C} / \mathrm{N}$ (Tabela 5). 
Em referência aos teores de $\mathrm{P}$, houve grande variação na sua distribuição nos diferentes sistemas de manejo nas profundidades estudadas (Tabela 5). A presença do P nos solos sob os SAF's pode ser atribuída, sobremaneira, ao contínuo aporte de resíduos orgânicos nos SAF's, como consequência da ação de sistemas radiculares diversificados, indicando maior eficiência na ciclagem de nutrientes (Tabela 5).

Embora o fósforo seja um nutriente essencial para o crescimento das plantas, ele se movimenta muito pouco na maioria dos solos e a ausência de revolvimento e a manutenção dos resíduos culturais na superfície do solo ocorridos em áreas sob SAF's contribuem para o aumento dos teores do elemento, principalmente na superfície, além da presença do sistema radicular das espécies arbóreas que favorece a presença do $\mathrm{P}$ em profundidade (Lopes, 1998; Falleiro et al., 2003).

Esses resultados condizem com os obtidos por Nogueira \& Oliveira (2008), que verificaram a influência do aporte contínuo de MOS em área sob SAF, proveniente dos estratos arbóreo, arbustivo e herbáceo, no aumento da concentração de P nesses sistemas, atribuído à elevada diversidade do material orgânico (Tabela 5). Para o período chuvoso se identificaram os maiores teores de P no solo sob agricultura tradicional de corte e queima, denotando efeito significativo da sazonalidade sobre os teores de P nesses sistemas. Os altos teores de P no solo sob ACQ podem ser atribuídos, segundo Falesi (1976); Cardoso et al. (1992); Silva \& Silva Júnior (2006), ao efeito da deposição das cinzas, decorrente da prática da queima e da mobilidade do nutriente pela chuva.

A eficiência doSAF13 com maior tempo de adoção em relação ao SAF6 para a maior conservação da qualidade química do solo denota maior equilíbrio desse sistema, calcado na diversidade dos seus componentes e no arranjo estrutural que o aproxima de uma área sob floresta (mata) nativa. Marin (2002) salienta que mudanças em variáveis do solo em razão do manejo, principalmente químicas, ocorrem em maior espaço de tempo sugerindo um tempo a partir de 10 anos para que sejam observadas maiores alterações.

\section{ConclusõES}

1. Os sistemas agroflorestais promoveram a melhoria dos indicadores químicos do solo calcada no aumento do $\mathrm{pH}$, redução da saturação por alumínio, aumento dos teores de nutrientes e maior estabilidade da qualidade química do solo sob efeito da sazonalidade.

2. O aporte de material orgânico e a grande biodiversidade dos sistemas agroflorestais aumentaram os teores de carbono e nitrogênio, garantindo maior permanência deste material e maiores efeitos benéficos promovidos pela matéria orgânica do solo.

3. A estrutura e a composição dos Sistemas Agroflorestais proporcionaram maior distribuição dos nutrientes do solo ao longo do perfil devido à estratificação das espécies e enraizamentos diferenciados.

\section{Agradecimentos}

Ao CNPq, pelo auxílio financeiro.

\section{LITERATURA CITADA}

Barreto, A. C.; Lima, F. H. S. Características químicas e físicas de um solo sob floresta, sistema agroflorestal e pastagem no sul da Bahia. Caatinga, v.19, p.415-425, 2006.

Bremner, J. M.; Mulvaney, C. S. Nitrogen total. In: Page, A. L.; Miller, R. H.; Keeney, D. R. (Ed.). Methods of soil analysis: chemical and microbiological properties. 2nd ed. Madison: American Society of Agronomy, 1982. v.2, p.595-624. Agronomy monograph, 9

Campanha, M. M.; Santos, R. H. S. Análise comparativa das características da serrapilheira e do solo em cafezais (Coffea arabica L.) cultivados em sistema agroflorestal e em monocultura na zona da mata-MG. Revista Árvore, v.31, p.805-812, 2007.

Cardoso, A.; Martins, P.F.S.; Veiga Jr., I. Solos de áreas ocupadas por pequenos agricultores em algumas localidades da microrregião de Marabá-PA. Pont à Pitre. Universidade des Antilas Guianas. p.101-123, 1992.

Cunha, E. Q.; Stone, L. F.; Ferreira, E. P. B.; Didonet, E. D.; Moreira, J. A. A. Atributos físicos, químicos e biológicos de solo sob produção orgânica impactados por sistema de cultivo. Revista Brasileira de Engenharia Agrícola e Ambiental, v.16, p.56-63, 2012.

D‘Andréa, A. F.; Silva, M. L. N.; Curi, N.; Siqueira, J. O.; Carneiro, M.A.C. Atributos biológicos indicadores da qualidade do solo em sistemas de manejo na região do Cerrado no sul do Estado de Goiás. Revista Brasileira de Ciências do Solo, v.26, p.913-923, 2002.

Delabie, J. H. C.; Jahyny, B.; Nascimento, I. C.; Mariano, S. F.; Lacau, S.; Campiolo, S. Contribution of cocoa plantations to the conservation of native ants (Insecta: Hymenoptera: Formicidae) with a special emphasis on the Atlantic forest fauna of southern Bahia, Brazil. Biodiversity Conservation, v.16, p.2359-2384, 2007.

Dias, H. C. T.; Figueira, M. D.; Silveira, V; Fontes, M. A. L.; Olveira-Filho, A. T. \& Scolforo, J. R. S. Variação temporal de nutrientes na serapilheira de um fragmento de floresta estacional semidecidual montana em Lavras, MG. Cerne, v.2, p.1-17, 2002.

Ehlers, W.; Kopke, V.; Hesse, F.; Bohm, W. Penetration resistance and root growth of oats tilled and untilled loess soil. Soil Tillage Research. v.3, p.261-275, 1983.

EMBRAPA - Empresa Brasileira de Pesquisa Agropecuária. Centro Nacional de Pesquisa de Solos. Manual de métodos de análise de solo. Rio de Janeiro: EMBRAPA, 1997. 21p.

EMBRAPA - Empresa Brasileira de Pesquisa Agropecuária Centro Nacional de Pesquisa de Solos. Sistema brasileiro de classificação de solos. 2.ed. Rio de Janeiro, 2006. 306p.

Falleiro, R. M. Souza, C. M.; Silva, C. S. W.; Sediyama, C. S.; Silva, A. A.; Fagundes, J. L. Influência dos sistemas de preparo nas propriedades químicas e físicas do solo. Revista Brasileira Ciência do Solo, v.27, p.1097-1104, 2003.

Falesi, I. C. Ecossistemas de pastagens cultivadas na Amazônia brasileira. Belém: Embrapa/CPATU, 1976. 193p. 
Fonte, S. J.; Barrios, E.; Six, J. Earthworm impacts on soil organic matter and fertilizer dynamics in tropical hillside agroecosystems of Honduras. Pedobiologia, v.53, p.327-335, 2010.

Franchini, J. C.; Gonzalez-Vila, F. J.; Cabrera, F.; Miyazawa, M.; Pavan, M.A. Rapid transformations of plant water-soluble organic compounds in relation to cation mobilization in an acid Oxisol. Plant Soil, v.31, p.55- 63, 2001.

Franzel, S.; Coe, R.; Cooper, P. Assessing the adoption potential of agroforestry practices in sub-Saharan Africa. Agricultural Systems, v.69, p.37-62, 2001.

Frazão, L. A.; Píccolo, M. C.; Feigl, B. J.; Cerri, C. C.; Cerri, C. E. P. Propriedades químicas de um Neossolo Quartzarênico sob diferentes sistemas de manejo no Cerrado mato-grossense. Pesquisa Agropecuária Brasileira, v.43, p.641-648, 2008.

Huerta, E.; Rodriguez-Olan, J.; Evia-Castillo, I.; MontejoMeneses, E.; Cruz- Mondragon, M.; Garcia-Hernandez, R.; Uribe, S. Earthworms and soil properties in Tabasco Mexico. European Journal Soil Biology, v.43, p.190-195, 2007.

Kaur, B.; Grupta, S. R.; Stingh, G. Soil carbon, microbial activity and nitrogen availability in Agroforestry sytems on moderately alkaline soils in northern India. Applied Soil Ecology, v.15, p.283-294, 2000.

Leite, L. F. C.; Mendonça, E. S. Estoques totais de carbono orgânico e seus compartimentos em argissolo sob floresta e sob milho cultivado com adubação mineral e orgânica. Revista Brasileira de Ciência do Solo, v.27, p.773-959, 2003.

Leite, L. F. C.; Oliveira, F. C.; Araujo, A. S. F.; Galvão, S. R. S.; Lemos, J. O; Silva, E. F. L. Soil organic carbon and biological indicators in an Acrisol under tillage systems and organic management in north-eastern Brazil. Australian Journal of Soil Research, v. 48, p. 258-265, 2010.

Lima, S. S. Impacto do manejo agroflorestal sobre a dinâmica de nutrientes e a macrofauna invertebrada nos compartimentos serapilheira-solo em área de transição no norte do Piauí/ Sandra Santana de Lima. Teresina: UFPI, 2008. Dissertação Mestrado

Lima, S. S.; Leite, L. F. C.; Aquino, A. M.; Oliveira, Francisco das Chagas ; Castro, A. A. J. F. Estoques da serrapilheira acumulada e teores de nutrientes em Argissolo sob manejo agroflorestal no norte do Piauí. Revista Árvore, v.34, p.75-84, 2010

Lima, S. S.; Leite, L. F. C.; Oliveira, F. C.; Costa, D. B. Atributos químicos e estoques de carbono e nitrogênio em Argissolo Vermelho-Amarelo sob sistemas agroflorestais e agricultura de corte e queima no norte do Piauí. Revista Árvore, v.35, p.51-60, 2011.

Lobato, F. A. O.; Andrade, E. M.; Meireles, A. C. M.; Santos, J. C. N.; Lopes, J. F. B. Perdas de solo e nutrientes em área de Caatinga decorrente de diferentes alturas pluviométricas. Revista Agroambiente, v.3, p.65-71, 2009.

Lopes, A. S. Manual internacional de fertilidade do solo. 2.ed. Piracicaba: Potafos, 1998. 177p.

Lopes, E. L. N.; Fernandes, A. R.; Grimaldi, C.; Ruivo, M. L. P.; Rodrigues, T. E.; Sarrazin, M.; Boletim do Museu Paraense Emílio Goeldi, Ciências Naturais 2006, v.1, p.127.

Maia, S. M. F.; Xavier, F. A. S. Frações de nitrogênio em Luvissolo sob sistemas agroflorestais e convencional no semi-árido cearense. Revista Brasileira de Ciência do Solo, v.32, p.381-392, 2008.
Maia, S. M. F.; Xavier, F. A. S.; Oliveira, T. S.; Mendonça, E. S.; Araújo Filho, J. A. Impactos de sistemas agroflorestais e convencional sobre a qualidade do solo no semi-árido cearense. Revista Árvore, v.30, p.837-848, 2006.

Marin, A. M. P. Impactos de um sistema agroflorestal com café na qualidade do solo. Viçosa: UFV, 2002. 83p. Tese Doutorado

McNeely, J. A.; Schroth, G. Agroforestry and biodiversity conservation - tradiotional practices, presents dynamics, and the lessons for the future. Biodiversity and Conservation, v.15, p.549-554, 2006.

Mendonza, H. N. S.; Lima E.; Anjos, L. H. C.; Silva, L. A.; Ceddia, M. B.; Antunes, M. V. M.Propriedades químicas e biológicas de solo de tabuleiro cultivado com cana-de-açúcar com e sem queima da palhada. Revista Brasileira de Ciência do Solo, v.24, p.201-207, 2000.

Moraes Sá, J. C; Cerri, C. C; Lal, R; Dick, W. A; Piccolo, M.C; Feigl, B. E. Soil organic carbon and fertility interactions affected by a tillage chronosequence in a Brazilian Oxisol. Soil \& Tillage Research, v.104, p.56-64, 2009.

Moreira, F. M. S.; Siqueira, J. O. Microbiologia e bioquímica do solo. 2.ed. Lavras: UFLA, 2006. 729p.

Mota, H.; Pequeno, P. L. L.; Brogio, M. P.; Shlindwein, J. A. In: Congresso Brasileiro de Ciência do solo, 2007, Gramado. Anais... Gramado: CBCS, 2007. CD Rom.

Moura, E. G. Agroambientes de transição avaliados numa perspectiva da agricultura familiar. In: Moura, E. G. (Ed.). Agroambientes de transição entre o trópico úmido e o semiárido do Brasil. São Luís: UEMA, 2004. cap. 1, p. 15-51.

Muzilli, O. Influência do sistema de plantio direto, comparado ao convencional, sobre a fertilidade da camada arável do solo. Revista Brasileira de Ciência do Solo, v.7, p.95-102, 1983.

Nair, P. K. R. Agroecosystem management in the 21st century: it is time for a paradigm shift. Journal of Tropical Agriculture, v.46, p.1-12, 2008.

Nair, P. K. R.; Kumar, B. M.; Nair, V. D. Agroforestry as a strategy for carbon sequestration. Journal of Plant Nutrition and Soil Science, p.10-23, 2009.

Nogueira, R. S; Oliveira, T. S. Formas de fósforo em Luvissolo Crómico Órtico sob sistemas agroflorestais no município de Sobral-CE. Ciência Agronômica, v.39, p.494-502, 2008.

Norgrove, L.; Csuzdi, C.; Forzi, F.; Canet, M.; Gounes, J. Shifts in soil faunal community structure in shaded cacao agroforests and consequences for ecosystem function in Central Africa. Tropical Ecology, v.50, p.71-78, 2009.

Oelbermann, M.; Voroney, R. P.; Thevathasan, N. V.; Gordon, A. M.; Kass, D. C. L.; Schlonvoigt, A. M. Soil carbon dynamics and residue stabilization in a Costa Rican and southern Canadian alley cropping system. Agroforestry System, v.68, p.27-36, 2006.

Pavinato, P. S. Disponibilidade de nutrientes no solo decomposição e liberação de compostos orgânicos de resíduos vegetais. Revista Brasileira de Ciência do Solo, v.32, p.911-920, 2008.

Peneireiro, F. M. Sistemas agroflorestais dirigidos pela sucessão natural: um estudo de caso. Piracicaba: ESALQ, 1999. 149p. Dissertação Mestrado 
Perin, E.; Ceretta, C. A.; Klamt, E. Tempo de uso agrícola e propriedades químicas de dois Latossolos do Planalto Médio do Rio Grande do Sul. Revista Brasileira de Ciência do Solo, v.27, p.665-674, 2003.

PLANAP - Plano de Ação para o Desenvolvimento Integrado da Bacia do Parnaíba: Atlas da Bacia do Parnaíba - Brasília: TDA Desenho \& Arte Ltda., 2006.126p.

Reitsma, R.; Parrish, J. D.; McLarney, W. The role of cacao plantations in maintaining forest avian diversity in southeastern Costa Rica. Agroforestry System, v.53, p.185193, 2001.

Saha, J. K.; Singh, A.B.; Ganheshamurty, A. N.; Kundu, S.; Biswas, A. K. Sulfur accumulation in vertsoil due to continuous gypsum application for six years and it effect on yield and biochemical constituents of soybean (Glicynne $\max$ L. Merrill). Journal of Plant Nutrition and Soil Science, v 164, p.317-320, 2001.

Salcedo, I.H.; Sampaio, E.V.S.B. Matéria orgânica do solo no bioma caatinga. In: Santos,G.S.; Silva, L.S.; Canellas, L.P.; Camargo, F.A.O. (eds). Fundamentos da matéria orgânica do solo: Ecossistemas tropicais e subtropicais. 2.ed. Porto Alegre: 2008, p.419-441.

Salmi, A. P.; Duerra, J. G. M.; Risso, J. A. M. Teores de nutrientes na biomassa aérea da leguminosa Flemingia macropylla. Revista Brasileira de Agroecologia, v.4, p.1013-1017, 2009.

Santos, A. F.; Menezes, R. S. C.; Fraga, V. S.; Pérez-Marin, A. M. Efeito residual da adubação orgânica sobre a produtividade de milho em sistema agroflorestal. Revista Brasileira de Engenharia Agrícola e Ambiental, v.14, p.12671272, 2010.

Santos, H. G.; Jacomine, P. K. T.; Anjos, L. H. C. dos; Oliveira, V. A de; Oliveira, J. B. de; Coelho, M. R.; Jumbreras, J. F.; Cunha, T. J. F. (ed.). Sistema brasileiro de classificação de solos. 2.ed. Rio de Janeiro: Embrapa Solos, 2006. 306p.

Schroth, G.; D’Angelo, S. A.; Teixeira, W. G.; Haag, D.; Lieberei, R. Conversion of secondary Forest into agroforestry and monoculture plantations in Amazônia: Consequences for biomass, litter and soil carbon stocks after 7 years. Forestry Ecology and Managenment, v.163, p.131-150, 2002.
Sharma, K. L.; Mandal, U. K.; Srinivas, K.; Vittal, K. P. R.; Mandal, B.; Grace, J. K.; Ramesh, V. Longterm soil management effects on crop yields and soil quality in a dryland Alfisol. Soil \& Tillage Research, v.83, p.246-259, 2005.

Silva, A. S; Silva, M. C. Prática de queimadas e as implicações sociais e ambientais na cidade de Araguaina-TO. Revista Caminhos de Geografia, v.18, p.8-16, 2006.

Silva, C. A.; Anderson, S. J.; Vale, F. R. Carbono, nitrogênio e enxofre em frações granulométricas de dois Latossolos submetidos a calagem e adubação fosfatada. Revista Brasileira de Ciência do Solo, v.23, p.593-602, 1999.

Silva, C. C.; Silveira, P. M. Influência de sistemas agrícolas em características químico-físicas do solo. Ciência e Agrotecnologia, v.26, p.505-515, 2002.

Silva, G. R.; Silva Júnior, M. L. Efeitos de diferentes usos da terra sobre as características químicas de um latossolo amarelo do Estado do Pará. Acta Amazonica, v.36, p.151158, 2006.

Smiley, G. L.; Kroschel, J. Temporal change in carbon stocks of cocoa-gliricidia agroforests in Central Sulawesi, Indonesia. Agroforestry System, v.73, p.219-231, 2008.

Sommer, R. Water and nutrient balance in deep soils under shifting cultivation with and without burning in the Eastern Amazon. Göttingen, Cuvillier, 2000. 240p. PhD thesis

Villatoro, M. A. A. Matéria orgânica e indicadores biológicos da qualidade do solo na cultura do café sob manejo agroflorestal e orgânico. Seropédica: UFRRJ, 2004. 176p. Tese Doutorado

Wastowski, A. D.; Rosa, G. M. Caracterização dos níveis de elementos químicos em solo, submetido a diferentes sistemas de uso e manejo, utilizando espectrometria de fluorescência de raios-x por energia dispersiva (EDXRF). Quimica Nova, v.33, p.1449-1452, 2010.

Yeomans, J. C.; Bremner, J. M. A rapid and precise method for routine etermimation of organic carbon in soil. Communications in Soil Science Plant Analysis, v.19, p.14671476, 1988. 